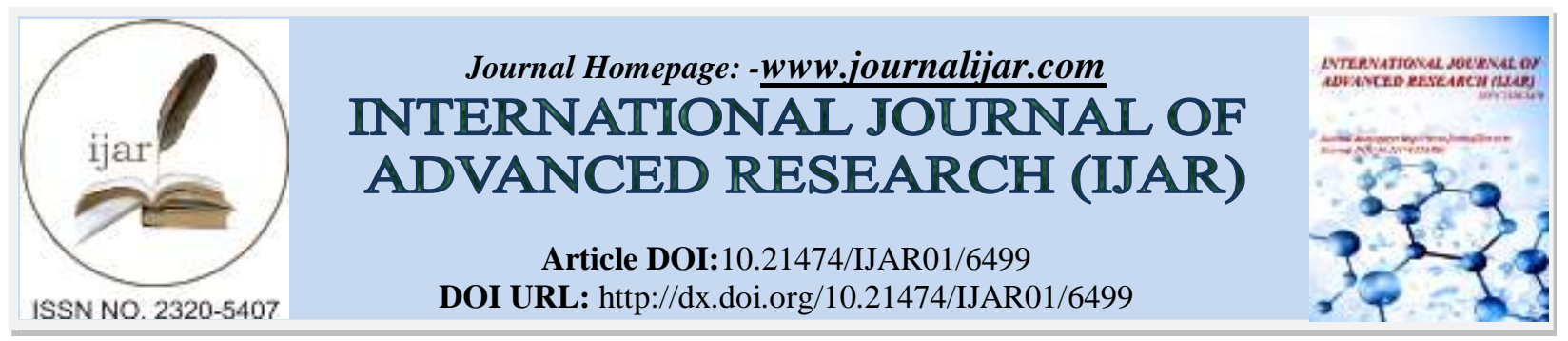

RESEARCH ARTICLE

\title{
HYPERTAURODONT: AN ENDODONTIC CHALLENGE TO DENTAL ANOMALY.
}

\section{Dr. Mayur Vaghasiya ${ }^{1}$, Dr. Jayasudha ${ }^{2}$, Dr. Shoba Fernandes ${ }^{3}$, Dr. Yash Bafna ${ }^{4}$, Dr. Kuldip ${ }^{5}$, Dr. Pooja Patel $^{6}$ and Dr. Jimisha Patel ${ }^{6}$.}

1. Post Graduate Student, Department of Pediatric and Preventive Dentistry, Narsinhbhai Patel Dental College and Hospital, Visnagar, India.

2. Professor, Department of Pediatric and Preventive Dentistry, Narsinhbhai Patel Dental College and Hospital, Visnagar, India.

3. Professor and Head, Department of Pediatric and Preventive Dentistry, Narsinhbhai Patel Dental College and Hospital, Visnagar, India.

4. Reader, Department of Pediatric and Preventive Dentistry, NPDCH, Narsinhbhai Patel Dental College and Hospital, Visnagar, India.

5. Senior lecturer, Department of Pediatric and Preventive Dentistry, Narsinhbhai Patel Dental College and Hospital, Visnagar, India.

6. Post Graduate Student, Department of Pediatric and Preventive Dentistry, Narsinhbhai Patel Dental College and Hospital, Visnagar, India.

\section{Manuscript Info}

Manuscript History

Received: 10 December 2017

Final Accepted: 12 January 2018

Published: February 2018

Keywords:-

Bull tooth, Enlarged pulp chamber,

Hypertaurodontism, Endodontic

treatment.

\section{Abstract}

Taurodontism is a rare dental anomaly in which the involved tooth has an enlarged and elongated body and pulp chamber with apical displacement of the pulpal floor. It is characterized by a deficiency in the constriction at the cemento enamel junction, with lengthened pulp chambers and apical displacement of the pulpal floor and gives the tooth a quadrilateral or cylindrical look. Taurodontism can also be seen in both the permanent and deciduous dentition, unilaterally or bilaterally and in any combination of teeth or quadrants. It can be association with several syndromes and abnormalities. Till date, most of dental practitioners are having little awareness about this clinical challenge while performing the endodontic therapy. Because of the challengingcomplexity and changed configuration in the tooth morphology, the critical need for its true diagnosis and management. This case report presents a case bilateral hypertaurodont of permanent maxillary first molars and their successful endodontic treatment.

Copy Right, IJAR, 2018,. All rights reserved.

\section{Introduction:-}

Dental morphological individuality are of particular importance in the study of population affinities and phylogenetic relationships (Constant \& Grine 2001). One of the most important dental anomaly in tooth morphology is taurodontism. Taurodontism is a morpho-anatomical change in the shape of the tooth in which the body of the tooth is enlarged and the roots are reduced in size and characterized by enlargement of the pulp chamber, which may approximate of the root apex, with the body of the tooth enlarged at the expense of the roots and apically displaced furcation areas. ${ }^{1}$ In 1908, Gorjanovic - Kramberger describes Taurodontism. ${ }^{2}$ In Kaprina, a 70,000 year old pre- 
Neanderthal fossil was discovered. ${ }^{3}$ Taurodontism was a frequent finding in early humans and is most common in Eskimos, possibly as a selective adaptation for cutting hide. ${ }^{4}$ The term 'taurodontism' was however first stated by Sir Arthur Keith in 1913. The origin of this term is from Greek "tauros" for "Bull" and "odontos" for "Tooth" which means 'bull tooth'.

The incidence of taurodontism has been reported to be different in modern man. The prevalence of taurodontism was reported to be $0.57-3.2 \%$ of white Americans, ${ }^{6,7} 8 \%$ of Jordanian, ${ }^{8}$ and $46.4 \%$ of young adult Chinese ${ }^{9}, 5.6 \%$ in Israeli people ${ }^{10}, 9.9 \%$ in normal Dutch $^{11}, 33-41 \%$ of certain Africans ${ }^{12}$ and $0.4 \%$ if North Indian population. ${ }^{13}$ Among the North Indian population Taurodontism were significantly more common in the maxilla $(65.6 \%)$ than in the mandible (34.4\%) and the maxillary second molar (34.4\%) was the most commonly involved tooth while maxillary first molar $(15.7 \%$ ) affected. According to the morphology hypotaurodonts were most common $(75 \%)$ and least common hypertaurodonts $(6.2 \%) .^{13}$

The etiology behind the taurodontism anomaly is supposed to be a primitive pattern, a mutation, an atavistic feature, a specialized or retrograde character, an X-linked trait, familial or an autosomal dominant trait as per various theories which is reported earlier. Various syndromes and genetic defects have shown their association with taurodontism but still, the real significance of it is not very clear. ${ }^{14}$ Taurodontism is caused by the failure of Hertwig's epithelial root sheath diaphragm to invaginate at the proper horizontal level (Hamner et al. 1964, Terezhalmy et al. 2001). Interference in the epitheliomesenchymatose induction has also been proposed as a possible etiology (Llamas \& Jimenez-Planas et al 1993).

In 1928 Taurodontism classified by Shaw, as hypotaurodontism, mesotaurodontism and hypertaurodontism based on the displacement of the floor of the pulp chamber. This subjective, arbitrary classification led normal teeth to be misdiagnosed as taurodontism. ${ }^{15}$ Feichfnger and Rossiwall in $1977,{ }^{16}$ stated that the distance from the bifurcation or trifurcation of the root to the cemento-enamel junction should be greater than the occluso-cervical distance for a bull tooth. In 1978, Shifman and Chanannel ${ }^{17}$ proposed a new classification and is the widely used system till now.

The external features and precise biometric methods are necessary in diagnosis of taurodontism. Taurodontism although not common, a preoperative radiograph serve as an important tool for diagnosing the taurodontic condition and helps in preventing unexpected challenges while performing successful endodontic therapy. ${ }^{18}$

Taurodontism is presents a challenge during negotiation, instrumentation and obturation in root canal therapy. Careful exploration of the grooves with magnification, ultrasonic irrigation, and a modified filling technique are during treatment of Taurodontic tooth .

This paper describes successful endodontic management of bilateral maxillary first molar with hypertaurodontism.

\section{Case Report:-}

A 11-year boy male patient reported to the Department of Pedodontics and Preventive Dentistry, Narsinhbhai Patel Dental College and Hospital, Visnagar, with pain in maxillary left and right posterior region since 13 days. Intraoral examination revealed deep caries with 16 and 26. However several times, he had experienced pain in his teeth while chewing food. The tooth was tender on percussion. Patient's medical history and family history was noncontributory. Upon vitality testing, The 16 and 26 showed no response to thermal and electric pulp testing which is indicate necrotic pulp.Intraoral periapical radiograph of 16 (Figure 1a) and 26 (Figure 2a)showed large pulp chamber extending beyond the cervical area reaching the furcation in the apical third region. Three short roots of taurodont teeth were seen at the furcation area in the apical third which is indicate taurodontism. Based on radiographic finding and taurodont index as proposed by Shiffman and Chanannel, The present case was hypertaurodontism in 16 and 26.

\section{Endodontic Management:-}

Under lidocaine (2\% with epinephrine 1:1, 00,000) local infiltration of maxillary left and right first molar, rubber dam and proper access opening made. The careful exploration revealed large pulp chamber, negotiation of the canals done, and it was found that three canals were originating deep into chamber. A total of $2.5 \%$ of sodium hypochlorite was used as irrigating solution using endodontic irrigation needle to prevent apical extrusion of sodium hypochloride. The furcation area was situated in the apical third region and four canal orifices were located at the furcation area; mesiobuccal, distobuccal and palatal in 16 and mesiobuccal, mesiolingual, distobuccal and palatal in 
26. The working lengths were estimated with a \#15 K file (Dentsply Maillefer; Ballaigues, Switzerland) by radiograph and then confirmed using electronic apex locator (Raypex 6, VDW). After working length determination the instrumentation of the canals was done to size 30 for the mesiobuccal and distobuccal canals, size 40 for the palatal canal in 16 and size 30 for the mesiobuccal and distobuccal canals, size 25 for the mesiolingual, size 40 for the palatal canal in 26 using RC prep (Premier, Ultradent, USA) as a lubricanting agent. A $2.5 \%$ of sodium hypochlorite and normal saline (sodium chloride injection I.P $0.9 \% \mathrm{w} / \mathrm{v}$ ) was alternatively used as irrigants at every change of instruments. The canals were dried with sterile paper points and were dressed with calcium hydroxide paste for 2 weeks. The access cavities were then temporarily sealed with intermediate restorative material.

After an uneventful inter-appointment gap, intermediate restorative material removed and normal saline used as a irrigant. After drying the canals with paper point, modified obturation technique was used because of complexity of inner root canal anatomy. The root canal walls were coated with selapex (SybronEndo,USA) and obturation done with gutta-percha cones (Densply Maillefer,Switzerland) using a traditional lateral compactionmethod. Remaining pulp chamber obturate with the thermoplasticized obturation technique (ULTRAFIL $®$ D, Coltene, Germany). The final radiograph confirmed a well-condensed filling of the four root canals and the tooth was restored with a Miracle mix (HI DENSE XP, SHOFU, Japan). After 7 days, tooth restored with preformed stainless steel crown (3M ESPE, US) of 16 (Figure 1b) and (Figure 2b).

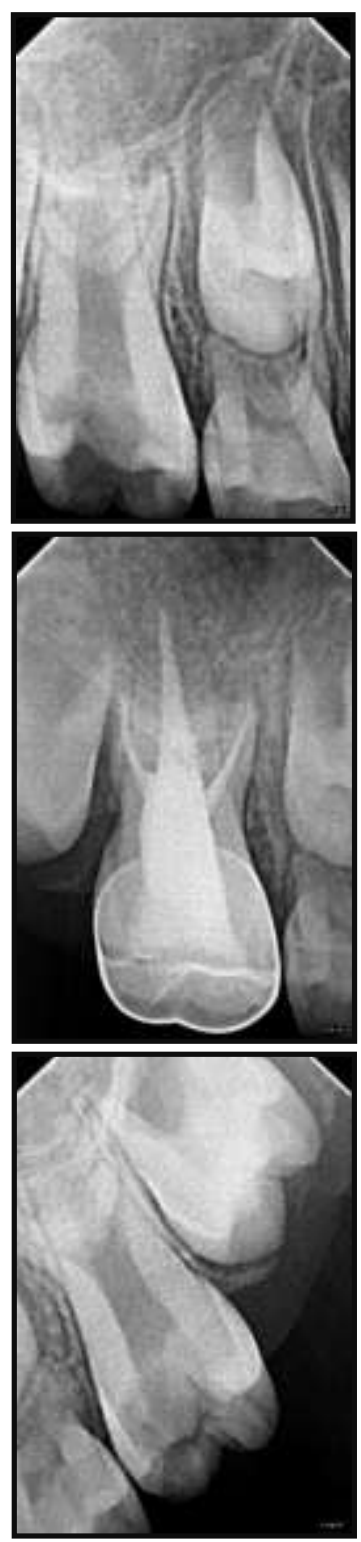

Figure 1a:- Pre-operative periapical radiograph (16)

Figure 1b:- Obturation of root canal with lateral condensation and thermoplasticized obturation (16)

Figure 2a:- Pre-operative periapical radiograph (26) 


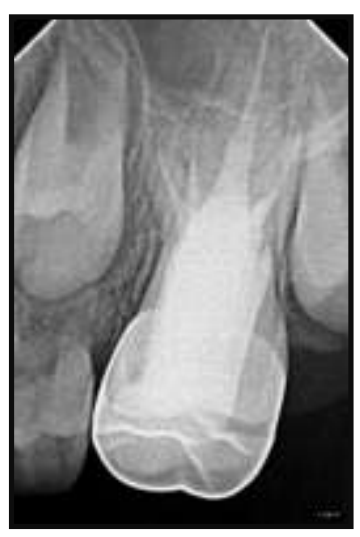

Figure 2b:- Obturation of root canal with lateral condensation and thermoplasticized obturation (26)

\section{Discussion:-}

Taurodontism is an anomaly of multi-rooted teeth which is characterized by enlargement of the pulp chamber. Widdowson defined taurodontism as the vertical deepening of pulp cavity at the expense of roots. ${ }^{18} \mathrm{~A}$ taurodont tooth shows wide variation in the size and shape of the pulp chamber, varying degrees of obliteration and canal configuration, apically positioned canal orifices, and the potential for additional root canal systems. ${ }^{19} \mathrm{Clinically}$, a taurodontic tooth appears to be a normal tooth. Therefore, a diagnosis of taurodontism is usually made from radiographic examination. There are differences in taurodontism in molar teeth, such as in the width of the pulp chambers and in the root canal anatomy. These differences make endodontic treatments more difficult in Taurodontism. ${ }^{20}$ Durr et al proposed that the morphology can affect the position of the orifices, making it more challenging to shape and fill the root canals. ${ }^{19}$

Taurodontism was at first thought to be a primitive tooth form (Moorrees 1957). On the other hand, it is found in such diverse groups as Inuit, Aleuts, Mongolians, Europeans, Scandinavians, African Americans, Chinese and white Americans amongst others (Moorrees 1957, Mjo“r 1972, Shifman \& Buchner 1976, MacDonald-Jankowski \& Li 1993, Goaz \& White 1994, Darwazeh et al. 1998, Backman \& Wahlin 2001, Tsesis et al. 2003). ${ }^{21}$ The reported rate of occurrence ranges from $0.57 \%$ to $4.37 \%$. Taurodontism appears most frequently as an isolated anomaly; it has also been associated with several syndromes and anomalies including Klinefelter's, Down's, tricho-dento-osseous syndrome and others. ${ }^{18}$

Varrela et al supported the concept that prevalence of taurodontism increases as the number of X-chromosomes increases and also indicate that expression of the triat and the number of X-chromosomes may be positively correlated. They have also suggested that X-chromosomal gene influencing development of enamel may also be involved in the development of taurodontism. ${ }^{22}$

The endodontic treatment in taurodont teeth has been described as complex and difficult. Tsesis et al, suggested that a combination of the cold lateral compaction technique and the warm vertical compaction technique can be used to obturate a taurodont tooth. ${ }^{23}$ Passive ultrasonic irrigation could be an important supplement for cleaning the root canal system, since, in comparison to traditional syringe irrigation, it can remove more organic tissue, planktonic bacteria, and dentin debris from the root canal. ${ }^{24}$

Periodontically, taurodont teeth may, in specific cases, offer favorable prognosis. Where periodontal pocketing or gingival recession occurs, the chances of furcation involvement are considerably less than those in normal teeth because taurodont teeth have to demonstrate significant periodontal destruction before furcation involvement occurs

Another endodontic challenge related to taurodontism is intentional replantation of tooth. The extraction of bull tooth is usually complicated because of dilated apical third. In contrast, it has also been hypothesized that because of its large body and little surface area of the taurodont tooth is embedded in the alveolus. This feature would make extraction much less difficult as long as roots are not widely divergent. ${ }^{22}$ Post placement for tooth reconstruction in taurodont tooth should be avoided. ${ }^{23}$ 


\section{Conclusion:-}

Taurodontism is a dental anamoly. A thorough knowledge of anatomic, etiology and radiographic features and its association with other syndromes of dental rarity should be well understood. From this case report we conclude that it isessential for a pediatric dentist to be well known with taurodontism not only with regards to clinical complications but also its management. Taurodontism also provides a valuable clue in detecting its relation with many syndromes and other systemic conditions.

\section{References:-}

1. Mena CA. Taurodontism. Oral Surg Oral Med Oral Pathol.1971; 32: 812-23.

2. Gorjanovic-Kramberger K, Uber prismatische Molarwurzeln rezenter and diluvialer Menschen. Anat Anz.1908; 32: 401-13.

3. Prakash R, Vishnu C, Suma B, Velmurugan N, Kandaswamy D. Endodontic management of taurodontic teeth. Indian J Dent Res.2005; 16:177-81.

4. Coon CS. Origin of races. Science.1963; 140: 208.

5. Witkop cj. Clinical aspects of dental anomalies. Int Dent J.1976; 26: 378-90.

6. Blumberg JE, Hylander WL, Goepp RA. Taurodontism:a biometric study. Am J Phys Anthropol.1971; 34: 243255.

7. Yeh SC, Hsu TY. Endodontic treatment in taurodontism with Klinefelter's syndrome: a case report. Oral Surg Oral Med Oral Pathol Oral Radiol Endod.1999; 88: 612-615.

8. Darwazeh AM, Hamasha AA, Pillai K. Prevalence of taurodontism in Jordanian dental patients. Dentomaxillofac Radiol.1998; 27: 3163-3165.

9. MacDonald-Jankowski DS, Li TT. Taurodontism in young adult Chinese population. Dentomaxillofac Radiol.1993; 22: 3140-3144.

10. Schiffman A, Chanannel I. Prevalence of taurodontism found in radiographic dental examination of 1200 young adult Israeli patients. Community Dent oral Epidemiol.1978; 6: 200-203.

11. Schalk - van der Weide Y, Steen WH, Bosman F. Taurodontism and length of teeth in patients with oligodontia. J Oral Rehábil.1993; 20(4): 401-412.

12. Shaw JCM. Taurodont teeth in South African races. J Anat.1928;62: 476-498.

13. Patil S, Doni B, Kaswan S, Rahman F.Prevalence of taurodontism in the North Indian population.J Clin Exp Dent.2013 Oct 1; 5(4): 179-82.

14. Bhat SS, Sargod S, Mohammed SV. Taurodontism in deciduous molars - A case report. J Indian Soc Pedod Prev Dent.2004; 22: 193-6.

15. Shaw JC. Taurodont Teeth in South African Races. J Anat.1928; 62:476-498.1.

16. Feichtinger C. Rosiwall B. Taurodontism in human sex chromosome aneuploidy. Arch Oral Biol.1977; 22: 3279.

17. Shifman A, Chanannel I. Prevalence of taurodontism found in radiographic dental examination of 1,200 young adult Israeli patients. Community Dent Oral Epidemiol.1978; 6: 200-3.

18. Lehtinen R. Taurodontism. A report of a case with familiar occurrence. Suom Hammaslaak Toim1971; 67: 713.

19. Durr DP, Campos CA, Ayers CS. Clinical significance of Taurodontism J Am Dent Assoc.1980; 100: 378-81.

20. Neslihan Simsek, Ali Keles, Mevlut Sinan Ocak. Endontic treatment of hypertaurodontism with multiple bilateral taurodontism. Journal of conservative dentistry.2013; Vol 16: Issue 5.

21. H. Jafarzadeh1, A. Azarpazhooh2 \& J. T. Mayhall. Taurodontism: a review of the condition and endodontic treatment challenges. International Endodontic Journal.2008; 41: 375-388.

22. Varrela J, Alvesalo L. Taurodontism in 47, XXY males: An effect of the extra X chromosome on root development. J Dent Res.1988; 67: 501-2.

23. Tsesis I, Shifman A, Kaufman AY. Taurodontism: An endodontic challenge. Report of a case. J Endod.2003; 29: 353-5.

24. Van der Sluis LW, Versluis M, Wu MK, Wesselink PR. Passive ultrasonic irrigation of the root canal: A review of the literature. Int Endod J.2007; 40: 415-26. 